\title{
Papel do letramento em saúde nos desfechos clínicos de idosos: uma revisão de
}

\section{escopo}

\author{
Role of health literacy in the clinical outcomes of the elderly: a scoping review \\ Papel de la alfabetización en salud en los resultados clínicos de los ancianos: una revisión del
}

alcance

Recebido: 23/08/2021 | Revisado: 02/09/2021 | Aceito: 06/09/2021 | Publicado: 07/09/2021

Francielle Brustolin de Lima Simch

ORCID: https://orcid.org/0000-0002-4722-4891 Universidade Federal do Paraná, Brasil E-mail: fbdlima@gmail.com

Sonia Mara de Andrade

ORCID: https://orcid.org/0000-0003-0247-9935 Universidade Federal do Paraná, Brasil E-mail: soniamaraandrade@gmail.com

Leonardo Martins Azeredo

ORCID: https://orcid.org/0000-0003-0169-6743 Universidade Federal do Paraná, Brasil E-mail: leonardomazeredo@gmail.com

Juliano Petry Pesarico

ORCID: https://orcid.org/0000-0002-6076-1317 Universidade Federal do Paraná, Brasil

E-mail: julianopetry@msn.com

\begin{abstract}
Resumo
O envelhecimento populacional é um processo natural, complexo e com diversas variáveis que atingem diretamente a qualidade de vida do idoso e da sociedade. Letramento em saúde é um conceito relativamente novo, que busca caracterizar a capacidade do indivíduo de compreender e ser agente ativo de seu processo saúde-doença. Dessa forma, objetivou-se verificar a relação entre o letramento em saúde e os desfechos clínicos apresentados pela população idosa. Utilizou-se o método de Revisão de Escopo segundo preconizado pelo Instituto Joanna Briggs. A coleta de dados foi realizada nas bases de dados Biblioteca Virtual de Saúde (BVS) e Pub Med. Utilizou-se os descritores "literacy", "letramento", "alfabetización en salud", "elderly", "aged", "idoso", "anciano", "clinical outcomes" e "health outcomes". A busca resultou na seleção de 19 artigos para a revisão, sendo verificado a relação do letramento em saúde com diversos desfechos clínicos, principalmente, hipertensão arterial sistêmica, diabetes mellitus, insuficiência cardíaca, risco cardiovascular, doença pulmonar obstrutiva crônica, admissões e hospitalizações, declínio cognitivo leve, doença de Alzheimer e depressão. Desfechos relacionados à dor e incapacidade não apresentaram relação com o letramento em saúde. A literatura demonstrou importante relação do letramento em saúde com diversos desfechos clínicos relevantes no contexto clínico do paciente, sendo fator valioso na avaliação clínica do idoso.
\end{abstract}

Palavras-chave: Letramento em saúde; Deterioração clínica; Idosos.

\begin{abstract}
Population aging is natural, complex and with variables that directly affect the quality of life of the elderly and society. Health literacy is a relatively new concept, which seeks to characterize the individual's ability to understand and be an active agent of their health-disease process. Thus, the aim was to verify the relationship between health literacy and clinical outcomes presented by the elderly population. The Scope Review method was used as recommended by the Joanna Briggs Institute. Data collection was performed in the databases Brazilian Virtual Health Library (BVS) and Pub Med. We used the descriptors "literacy", "letramento", "alfabetización en salud", "elderly", "aged", "idoso", "anciano", "clinical outcomes" and "health outcomes". The search resulted in the selection of 19 articles for the review, being verified the relationship of health literacy with various clinical outcomes, principally, systemic arterial hypertension, diabetes mellitus, heart failure, cardiovascular risk, chronic obstructive pulmonary disease, admissions and hospitalizations mild cognitive impairment, Alzheimer's disease and depression. Outcomes related to pain and disability were not related to health literacy. The literature has pointed out an important relationship between health literacy and several relevant clinical outcomes in the patient's clinical context, being a valuable factor in the clinical evaluation of the elderly.
\end{abstract}

Keywords: Health literacy; Clinical deterioration; Aged. 


\begin{abstract}
Resumen
El envejecimiento de la población es un proceso natural, complejo y con varias variables que inciden directamente en la calidad de vida de las personas mayores y la sociedad. La alfabetización en salud es un concepto relativamente nuevo, que busca caracterizar la capacidad del individuo para comprender y ser un agente activo de su proceso saludenfermedad. De esa forma, el objetivo fue verificar la relación entre la alfabetización en salud y los resultados clínicos presentados por la población anciana. El método revisión del alcance se utilizó según lo recomendado por el Instituto Joanna Briggs. La recolección de datos se realizó en las bases de datos Biblioteca Virtual de Salud (BVS) y Pub Med. Usamos los descriptores "literacy", "letramento", "alfabetización en salud", "elderly", "aged", "idoso", "anciano", "clinical outcomes" y "health outcomes". La búsqueda resultó en la selección de 19 artículos para la revisión, verificándose la relación de alfabetización en salud con diversos resultados clínicos, principalmente, hipertensión arterial sistémica, diabetes mellitus, insuficiencia cardíaca, riesgo cardiovascular, enfermedad pulmonar obstructiva crónica, ingresos y hospitalizaciones, deterioro cognitivo leve, enfermedad de Alzheimer y depresión. Los resultados relacionados con el dolor y la discapacidad no se relacionaron con la alfabetización en salud. La literatura ha señalado una relación importante entre la alfabetización en salud y varios resultados clínicos relevantes en el contexto clínico del paciente, siendo un factor valioso en la evaluación clínica de los ancianos.
\end{abstract}

Palabras clave: Alfabetización en salud; Deterioro clínico; Anciano.

\title{
1. Introdução
}

Com o progressivo envelhecimento da população brasileira e a concomitante mudança demográfica mundial, tornouse cada vez mais importante considerar, além da expectativa de vida da população, a sua autonomia e qualidade de vida, levando em consideração as implicações acarretadas pelo processo de envelhecimento (Massi et al. 2010). Segundo a Organização das Nações Unidas (ONU), no ano de 2015, a população de idosos no Brasil, representava 11,7\% da população brasileira; para o futuro, as Nações Unidas projetam que no ano de 2039 esse número dobrará, chegando a 23,5\% da população (IBGE, 2016).

A senescência na população ocorre de forma desigual, particular e irreversível, naturalmente acarreta alterações físicas, psicológicas e comportamentais; podendo ser determinada por fatores genéticos, fatores ambientais e hábitos pregressos e atuais de vida; sendo então caracterizada por redução das capacidades físicas ou cognitivas, além de uma redução funcional normal (Moreira et al. 2020).

O avançar da idade, por si só, não leva a perda de funções indispensáveis para a conservação de uma vida funcional e feliz; pois as mudanças fisiológicas causadas pelo envelhecimento, principalmente no sistema nervoso, respiratório e locomotor, não impedem que o indivíduo mantenha sua atuação na sociedade com qualidade, mesmo com certas limitações; portanto, a diferenciação de alterações fisiológicas inatas do envelhecimento, de alterações pouco relacionadas a idade, é de grande importância para o manejo multidimensional do idoso (Morais, 2012).

O processo de envelhecimento populacional trouxe novos desafios para a sociedade; requisitando do poder público, políticas de enfrentamento aos determinantes sociais da saúde, entre eles: renda, estrutura familiar e educação; dessa forma, buscando eliminar a causa primária das doenças mais frequentes na terceira idade (Geib, 2012).

As dificuldades de comunicação na relação médico-paciente, podem trazer obstáculos na tomada de decisões quanto às estratégias de manejo da saúde do paciente idoso, pois a dificuldade de compreensão das informações sobre seus agravos de saúde tem grande impacto na prática do autocuidado e da adesão às propostas terapêuticas (Neto et al. 2019).

A linguagem tem papel importante no envelhecimento, permitindo maior integração do idoso com a sociedade, mantendo sua identidade de cidadão (Filho \& Massi, 2014). Letramento em saúde (LS) representa a capacidade do paciente em identificar e compreender as informações necessárias para a tomada de decisões quanto a sua saúde, sendo fator importante para a identificação dos aspectos do autocuidado e das ações do sistema de saúde (Santos, Portella, Scortegagna \& Santos, 2015).

O LS é um termo recente no sistema de saúde brasileiro, sendo indicada sua utilização na saúde para a melhora dos cuidados e promoção de saúde na sociedade, por ser um conceito complexo, possui múltiplos fatores relacionados, sendo 
interligado a condições sociais e ambientais; dessa forma, quando bem disperso e enraizado na população, tem a capacidade de tornar o idoso, o agente das decisões sobre sua saúde (Santos \& Portella, 2016).

A capacidade de obter, compreender, refletir e aplicar as propostas terapêuticas para cuidado em saúde, é a verbalização do conceito de LS; sua definição está baseada na percepção do paciente quanto às suas dificuldades nos cuidados com sua saúde (Marques, Escarce \& Lemos, 2018).

O nível de escolaridade não é um indicador fidedigno do LS, dessa forma, instrumentos específicos para sua mensuração fazem-se necessários, o Test of Functional Health Literacy in Adults - TOFHLA (Parker et al., 1995) foi desenvolvido como um método de avaliação do LS na língua inglesa e testa a capacidade de leitura e compreensão de texto e compreensão numérica de materiais relacionados ao contexto da saúde, ele não é o único instrumento disponível para avaliação do LS, sendo o REALM (Estimativa Rápida de Alfabetização de Adultos em Medicina) validado por Murphy et al. (1993) também um dos mais utilizados, o qual avalia instruções relacionadas ao uso de medicamentos e a compreensão da terapêutica por parte dos usuários, a versão completa do instrumento está disponível apenas em inglês. No Brasil, o S-TOFHLA (Baker et al., 1999), versão reduzida do TOFHLA, foi validado e traduzido por Carthery-Goulart et al. (2009), e engloba 36 itens de leitura e 4 itens de habilidades numéricas (Baker et al., 1999). O instrumento apresenta pontuação que varia de zero a 100, indivíduos que obtém menos 53 pontos são designados como LS inadequado, indivíduos que obtém entre 54 e 66 pontos são designados como LS marginal, e indivíduos que obtém mais de 67 pontos possuem LS adequado (Carthery-Goulart et al. 2009).

Na população brasileira, os idosos são os mais influenciados pelo LS inadequado, devido a sua vulnerabilidade social e ao processo natural de senescência (Lima et al. 2019). O letramento em saúde adequado, tende a conduzir o indivíduo para uma melhor condição de saúde, uma melhor compreensão das propostas terapêuticas e das medidas de controle e prevenção de agravos (Neto et al. 2019). A adesão do paciente aos manejos clínicos é diretamente proporcional ao seu nível de letramento em saúde, tornando-se indispensável aos profissionais da saúde, a identificação da capacidade de entendimento do paciente por meio dos instrumentos de mensuração do LS, dessa forma, realizar intervenções que propiciem ao paciente melhor entendimento e senso crítico quanto a necessidade do tratamento empregado a sua condição de saúde (Maragno \& Luiz, 2016).

Nessa perspectiva, por meio da metodologia de revisão de escopo, o presente estudo tem como objetivo identificar as relações do letramento em saúde com os desfechos clínicos da população idosa.

\section{Metodologia}

Revisões de escopo tem como linha guia, o mapeamento de conceitos-chave disponíveis na literatura; seu objetivo é verificar a dimensão das evidências, explorando, mapeando e resumindo as informações obtidas por pesquisas pregressas. Sendo a revisão de escopo, uma ótima forma de examinar temas emergentes (The Joanna Briggs Institute, 2021).

Esta pesquisa está delineada como uma revisão de escopo, seguindo os métodos propostos pelo Instituto Joanna Briggs (JBI) (The Joanna Briggs Institute, 2021). A estrutura do desenvolvimento da pesquisa seguiu o seguinte ordenamento: 1. Identificação da questão de pesquisa; 2. Identificação da estratégia de busca; 3. Seleção dos estudos; 4. Extração dos dados; 5. Análise das evidências e 6. Apresentação dos resultados (The Joanna Briggs Institute, 2021).

Para a identificação da pergunta norteadora da pesquisa, utilizou-se a estratégia Participantes (P), Conceito (C), Contexto (C), (PCC) (The Joanna Briggs Institute, 2021), enquadrando a temática do estudo no seguinte arranjo: P - Idosos; C - Letramento em saúde; C - Desfechos em saúde. Dessa forma, obtendo a pergunta norteadora estruturada da seguinte forma: quais as relações entre os desfechos clínicos de idosos e o seu letramento em saúde?

Utilizou-se a Biblioteca Virtual de Saúde (BVS) e a base de dados Pub Med como fontes para pesquisa bibliográfica durante os meses de janeiro e fevereiro de 2021. Utilizou-se restrição de tempo de cinco anos na busca dos artigos, do primeiro 
dia de janeiro de 2016 ao primeiro dia de janeiro de 2021. A seleção e análise dos artigos ocorreu de forma pareada, sendo realizada por dois pesquisadores. As inconsistências e dúvidas originadas durante essa etapa foram analisadas por todos os autores e solucionadas por consenso.

A definição dos descritores foi auxiliada pela plataforma Descritores em Ciências da Saúde (DeCS) e definida segundo as particularidades de cada plataforma, utilizando os termos-chave da pergunta norteadora, definimos os seguintes descritores para a busca: "literacy", "letramento", "alfabetización en salud", “elderly", "aged”, "idoso", "anciano", "clinical outcomes" e "health outcomes", utilizando os booleanos "AND" e "OR" para relacionar os descritores.

Todos os artigos identificados nas plataformas bibliográficas que responderam à pergunta norteadora, estavam disponíveis em língua portuguesa, inglesa ou espanhola, pesquisas qualitativas ou quantitativas e que abordassem a população maior de 65 anos foram incluídos nesta pesquisa. Os artigos que não responderam à pergunta norteadora, apresentavam-se em duplicatas nas plataformas de dados, não se encontravam no público alvo maior de 65 anos, livros, revisões sistemáticas, teses, cartas, dissertações e disponíveis em línguas diferentes do português, inglês ou espanhol, foram excluídas durante a seleção.

A seleção dos trabalhos ocorreu através do decremento dos artigos disponíveis nas bases de dados; primeiramente os artigos foram avaliados pelo conteúdo dos seus títulos e palavras-chave, posteriormente realizou-se a avaliação de seus resumos e adiante realizou-se a leitura total, na íntegra, dos artigos selecionados. As referências presentes nos artigos lidos na íntegra foram analisadas, em uma nova busca por trabalhos que se enquadram nos critérios desta revisão. Todos os artigos selecionados tiveram suas informações extraídas, analisadas e resumidas através de um instrumento com os seguintes itens: autor principal, ano, base de dados, título, local do estudo, tipo de estudo, população do estudo, tamanho da amostra, instrumento de avaliação do letramento em saúde, resultados e resposta à questão norteadora da pesquisa.

\section{Resultados}

A busca nas bases de dados utilizadas forneceu um total de 610 artigos, sendo 576 disponíveis no PubMed e 34 disponíveis na Biblioteca Virtual de Saúde (BVS). Com a seleção dos trabalhos, foram excluídos 571 artigos através da leitura do título e/ou resumo. Para a leitura na íntegra foram selecionados 39 trabalhos, a avaliação das referências desses 39 artigos resultou na inclusão de mais cinco artigos. Do total de 44 artigos avaliados na íntegra, 24 foram excluídos por não responderem à pergunta de pesquisa e um foi excluído por não fornecer todos os dados necessários para a completa avaliação.

Esta revisão selecionou o total de 19 artigos (Figura 1) para sua elaboração. As informações sobre o fluxo do processo de análise dos trabalhos identificados nas bases de dados estão esquematizadas na Figura 1 conforme recomendação do JBI, seguindo o checklist do Preferred Report Items for Systematic reviews and Meta-Analyses extension for Scoping Reviews (PRISMA-ScR) (The Joanna Briggs Institute, 2021). 
Figura 1 - Fluxo do processo de análise dos artigos, segundo PRISMA-ScR.

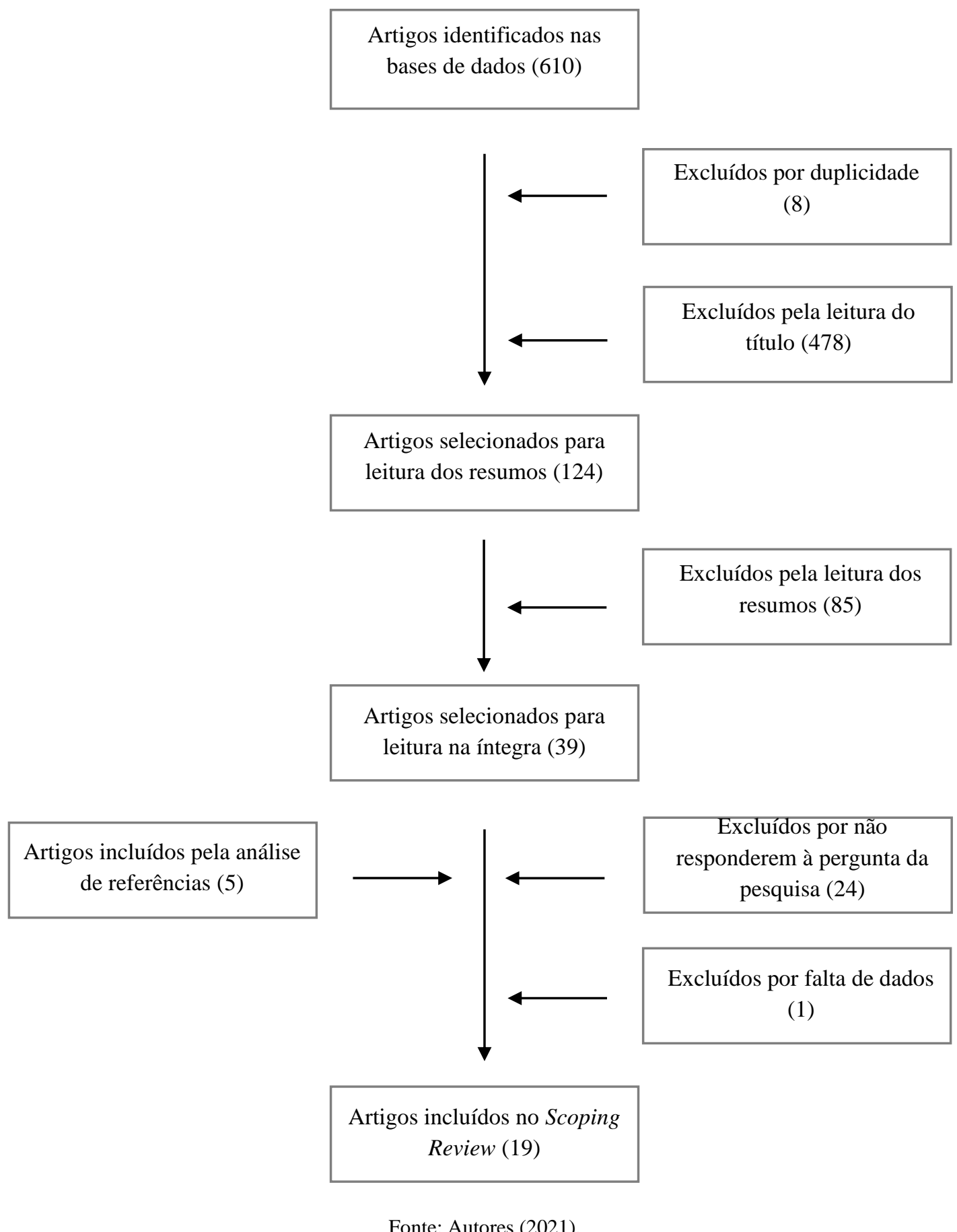

Todos os 19 estudos apresentados nesta revisão de escopo foram publicados entre o ano de 2016 (Santos \& Portela, 2016; Punte-Maestu et al. 2016; Wannasirikul, Termsirikulchai, Sujirarat, Benjakul \& Tanasugarn, 2016; Cox et al. 2016) e o ano de 2020 (Souza, Farfel, Jaluul, Queiroz \& Nery, 2020; Do et al. 2020; Finbraten et al. 2020). Dos trabalhos selecionados, segundo seus tipos de estudo, foram identificados nove coortes (Punte-Maestu et al. 2016; Fabbri et al. 2018; León-González et al. 2017; Yu, Wilson, Han et al. 2017; Shi et al. 2017; Lamar et al. 2018; Cox et al. 2016; Wilson, Yu James, Bennett \& Boyle, 2017; Yu, Wilson, Schneider, Bennett \& Boyle, 2017), um ecológico (Santos \& Portella, 2016) e nove estudos transversais (Costa, Costa, Nakano, Apolinário \& Santana, 2019; Souza et al. 2020; Como, 2018; Fernández-Silva, Alonso- 
González, Gonzáles-Pérez, Gestal-Otero \& Díaz-Grávalos, 2018; Murtaugh et al. 2017; Do et al, 2020; Finbraten et al. 2020; Liu, Xue, Xue \& Hou, 2018; Wannasirikul et al. 2016). Todos os trabalhos tinham como objetivo identificar a relação do letramento em saúde com algum desfecho clínico investigado, conforme informações apresentadas no Quadro 1.

Quadro 1 - Informações dos trabalhos incluídos na revisão de escopo, segundo o autor principal e ano de publicação, tipo de estudo e resposta à pergunta norteadora.

\begin{tabular}{|c|c|c|c|}
\hline $\begin{array}{l}\text { Autor principal e ano } \\
\text { de publicação. }\end{array}$ & $\begin{array}{l}\text { Tipo de } \\
\text { estudo }\end{array}$ & $\begin{array}{l}\text { Local mostra e idade } \\
\text { média }\end{array}$ & Resposta à pergunta norteadora \\
\hline Do et al. 2020. & Transversal & $\begin{array}{l}\text { Local: Vietnã. } \\
\text { Amostra: } 928 \\
\text { participantes. } \\
\text { Idade média: } 68,2 \text { anos. }\end{array}$ & $\begin{array}{l}\text { Objetivo: Estudo avaliou a relação entre o letramento em saúde e } \\
\text { comportamentos em saúde e depressão em pacientes com covid-19. } \\
\text { Desfechos: Elevadas pontuações em letramento em saúde foram } \\
\text { associadas a menor probabilidade de depressão. }\end{array}$ \\
\hline Finbraten et al. 2020. & Transversal & $\begin{array}{l}\text { Local: Noruega. } \\
\text { Amostra: } 388 \\
\text { participantes. } \\
\text { Idade média: } 73 \text { anos. }\end{array}$ & $\begin{array}{l}\text { Objetivo: Estudo avaliou a relação entre o letramento em saúde, } \\
\text { diabetes mellitus tipo } 2 \text { e comportamentos em saúde. } \\
\begin{array}{l}\text { Desfecho. Letramento em saúde não foi associado ao controle } \\
\text { glicêmico, entretanto foi associado a melhores condições gerais de } \\
\text { saúde. }\end{array}\end{array}$ \\
\hline Souza et al. 2020. & Transversal & $\begin{array}{l}\text { Local: Brasil } \\
\text { Amostra: } 166 \\
\text { participantes. } \\
\text { Idade média: } 68 \text { anos. }\end{array}$ & $\begin{array}{l}\text { Objetivo: Estudo avaliou a relação do letramento em saúde com o } \\
\text { controle glicêmico em portadores de diabetes mellitus } 2 \text { (DM2). } \\
\text { Desfecho: Pontuação na escala de letramento em saúde foi } \\
\text { associada a valores da HbA1c. Letramento em saúde foi associado } \\
\text { ao nível de controle glicêmico. }\end{array}$ \\
\hline Costa et al. 2019. & Transversal & $\begin{array}{l}\text { Local: Brasil. } \\
\text { Amostra: } 392 \\
\text { participantes. } \\
\text { Idade média: } 68,64 \text { anos }\end{array}$ & $\begin{array}{l}\text { Objetivo: Estudo avaliou a relação do letramento em saúde com o } \\
\text { controle da pressão arterial sistêmica (PAS) em pacientes } \\
\text { hipertensos. } \\
\begin{array}{l}\text { Desfecho: Letramento em saúde inadequado foi associado ao } \\
\text { controle inadequado da PAS. }\end{array}\end{array}$ \\
\hline Como, 2018. & Transversal & $\begin{array}{l}\text { Local: EUA. } \\
\text { Amostra: } 175 \\
\text { participantes. } \\
\text { Idade média: } 73,6 \text { anos. }\end{array}$ & $\begin{array}{l}\text { Objetivo: Estudo avaliou a relação do letramento em saúde com } \\
\text { insuficiência cardíaca crônica. } \\
\text { Desfecho: Não foi encontrada relação entre o letramento em saúde } \\
\text { e adesão à medicação, estado de saúde físico e estado de saúde } \\
\text { mental. }\end{array}$ \\
\hline Fabbri et al. 2018. & Coorte & $\begin{array}{l}\text { Local: EUA. } \\
\text { Amostra: } 2487 \\
\text { participantes. } \\
\text { Idade média: } 73,5 \text { anos. }\end{array}$ & $\begin{array}{l}\text { Objetivo: Estudo avaliou a associação do letramento em saúde com } \\
\text { desfechos em pacientes com insuficiência cardíaca. } \\
\text { Desfecho: Baixo letramento em saúde foi associado a risco } \\
\text { aumentado de morte e número médio mais alto de internações } \\
\text { hospitalares. }\end{array}$ \\
\hline $\begin{array}{l}\text { Fernández-Silva et al. } \\
2018 .\end{array}$ & Transversal & $\begin{array}{l}\text { Local: Espanha. } \\
\text { Amostra: } 103 \\
\text { participantes. } \\
\text { Idade média: } 65,4 \text { anos. }\end{array}$ & $\begin{array}{l}\text { Objetivo: Estudo avaliou a associação do letramento em saúde e a } \\
\text { diabetes mellitus tipo } 2 \text { (DM2). } \\
\text { Desfechos: Encontrada relação entre o grau de controle da doença } \\
\text { diabética e o grau de letramento em saúde. }\end{array}$ \\
\hline Lamar et al. 2018. & Coorte & $\begin{array}{l}\text { Local: EUA. } \\
\text { Amostra: } 908 \\
\text { participantes. } \\
\text { Idade média: } 81 \text { anos. }\end{array}$ & $\begin{array}{l}\text { Objetivo: Estudo avaliou a associação do letramento em saúde com } \\
\text { indicadores da diabetes. } \\
\text { Desfechos: Menor nível de alfabetização em saúde foi associado a } \\
\text { níveis mais altos de hemoglobina glicada e níveis mais altos de } \\
\text { glicose. }\end{array}$ \\
\hline Liu et al. 2018. & Transversal & $\begin{array}{l}\text { Local: China. } \\
\text { Amostra: } 1396 \\
\text { participantes. } \\
\text { Idade média: } 77,37 \text { anos. }\end{array}$ & $\begin{array}{l}\text { Objetivo: Estudo avaliou a associação entre o letramento em saúde } \\
\text { e saúde física, mental e atividade da vida diária. } \\
\text { Desfechos: Letramento em saúde foi associado à saúde física e não } \\
\text { foi associado à saúde mental. Baixo letramento em saúde foi } \\
\text { associado a piores condições de vida. }\end{array}$ \\
\hline
\end{tabular}




\begin{tabular}{|c|c|c|c|}
\hline $\begin{array}{l}\text { León-González et al. } \\
2017 .\end{array}$ & Coorte & $\begin{array}{l}\text { Local: Espanha. } \\
\text { Amostra: } 556 \\
\text { participantes. } \\
\text { Idade média: } 85,6 \text { anos. }\end{array}$ & $\begin{array}{l}\text { Objetivo: Estudo avaliou a relação do letramento em saúde com } \\
\text { desfechos em pacientes com insuficiência cardíaca. } \\
\text { Desfecho: Letramento em saúde alto foi associado a menor grau de } \\
\text { comprometimento cardíaco e menor comprometimento cognitivo. } \\
\text { Não houve associação entre o letramento em saúde e causas de } \\
\text { mortalidade. }\end{array}$ \\
\hline Murtaugh et al. 2017 & Transversal & $\begin{array}{l}\text { Local: EUA. } \\
\text { Amostra: } 588 \\
\text { participantes. } \\
\text { Idade média: } 73 \text { anos. }\end{array}$ & $\begin{array}{l}\text { Objetivo: Estudo avaliou a associação entre a intensidade da dor e o } \\
\text { letramento em saúde. } \\
\text { Desfecho: Não foi encontrada relação entre o letramento em saúde } \\
\text { e a intensidade da dor. Também não houve relação entre a } \\
\text { incapacidade relacionada a dor e o letramento em saúde. }\end{array}$ \\
\hline Shi et al. 2017. & Coorte & $\begin{array}{l}\text { Local: China. } \\
\text { Amostra: } 360 \\
\text { participantes. } \\
\text { Idade média: } 66,6 \text { anos. }\end{array}$ & $\begin{array}{l}\text { Objetivo: Estudo avaliou a relação entre o letramento em saúde e a } \\
\text { hipertensão arterial sistêmica (HAS). } \\
\text { Desfecho: Nível de letramento em saúde foi associado a pressão } \\
\text { arterial sistêmica (PAS). PAS elevado indicou menor letramento } \\
\text { em saúde. O uso regular da medicação foi associado ao letramento } \\
\text { em saúde. Baixo letramento em saúde foi associado a maior risco } \\
\text { cardiovascular. }\end{array}$ \\
\hline Wilson et al. 2017. & Coorte & $\begin{array}{l}\text { Local: EUA. } \\
\text { Amostra: } 755 \\
\text { participantes. } \\
\text { Idade média: } 81,7 \text { anos. }\end{array}$ & $\begin{array}{l}\text { Objetivo: Estudo avaliou a associação entre o letramento em saúde } \\
\text { e a saúde cognitiva. } \\
\text { Desfecho: Letramento em saúde elevado foi associado a diminuição } \\
\text { do risco de doença de Alzheimer. Nível elevado de letramento em } \\
\text { saúde foi associado a nível de cognição mais elevado e menor } \\
\text { declínio cognitivo. }\end{array}$ \\
\hline $\begin{array}{l}\text { Yu, Wilson, Han et al. } \\
2017 \text {. }\end{array}$ & Coorte & $\begin{array}{l}\text { Local: EUA. } \\
\text { Amostra: } 799 \\
\text { participantes. } \\
\text { Idade média: } 81,7 \text { anos. }\end{array}$ & $\begin{array}{l}\text { Objetivo: Estudo avaliou a relação do letramento em saúde com a } \\
\text { demência por doença de Alzheimer (DA). } \\
\text { Desfecho: Declínio acentuado do letramento em saúde foi } \\
\text { associado a maior risco de demência por DA e a maior risco de } \\
\text { comprometimento cognitivo leve. }\end{array}$ \\
\hline $\begin{array}{l}\text { Yu, Wilson, Schneider } \\
\text { et al. } 2017 \text {. }\end{array}$ & Coorte & $\begin{array}{l}\text { Local: EUA. } \\
\text { Amostra: } 805 \\
\text { participantes. } \\
\text { Idade média: } 81,5 \text { anos. }\end{array}$ & $\begin{array}{l}\text { Objetivo: Estudo avaliou a associação entre letramento em saúde e } \\
\text { a demência por doença de Alzheimer. } \\
\text { Desfecho: Menor letramento em saúde foi associado a maior risco } \\
\text { para desenvolvimento de demência por doença de Alzheimer. }\end{array}$ \\
\hline Cox et al. 2016. & Coorte & $\begin{array}{l}\text { Local: EUA. } \\
\text { Amostra: } 264 \\
\text { participantes. } \\
\text { Idade média: } 66,6 \text { anos. }\end{array}$ & $\begin{array}{l}\text { Objetivo: Estudo avaliou a associação entre o letramento em saúde } \\
\text { e o uso de assistência de saúde hospitalar em pacientes com } \\
\text { insuficiência cardíaca. } \\
\text { Desfecho: Nível de letramento em saúde baixo foi associado a } \\
\text { maiores usos de cuidados em saúde. Nível baixo também foi } \\
\text { associado a maiores usos não planejados de cuidados de saúde. }\end{array}$ \\
\hline $\begin{array}{l}\text { Santos \& Portella, } \\
2016 .\end{array}$ & Ecológico & $\begin{array}{l}\text { Local: Brasil. } \\
\text { Amostra: } 114 \\
\text { participantes. } \\
\text { Idade média: } 67,4 \text { anos. }\end{array}$ & $\begin{array}{l}\text { Objetivo: Estudo avaliou a relação do letramento em saúde com } \\
\text { desfechos relacionados a diabetes. } \\
\text { Desfecho: Baixo letramento em saúde foi associado a complicações } \\
\text { clínicas presentes na diabetes, como complicações relativas à } \\
\text { função visual e doença renal. }\end{array}$ \\
\hline $\begin{array}{l}\text { Puente-Maestu et al. } \\
2016\end{array}$ & Coorte & $\begin{array}{l}\text { Local: Espanha } \\
\text { Amostra: } 296 \\
\text { participantes. } \\
\text { Idade média: } 68 \text { anos. }\end{array}$ & $\begin{array}{l}\text { Objetivo: Estudo avaliou a associação do letramento em saúde com } \\
\text { a doença pulmonar obstrutiva crônica (DPOC). } \\
\text { Desfecho: Pacientes com DPOC e letramento em saúde inadequado } \\
\text { necessitam de maior assistência de terceiros. Letramento em saúde } \\
\text { inadequado também foi associado a maior incidência de ansiedade } \\
\text { e depressão. Letramento em saúde inadequado aumentou a chance } \\
\text { de admissões ou visitas ao departamento de emergência. Verificou- } \\
\text { se associação entre o letramento em saúde inadequado e maior } \\
\text { morbimortalidade, mais admissões hospitalares e pior expectativa } \\
\text { de vida. }\end{array}$ \\
\hline $\begin{array}{l}\text { Wannasirikul et al. } \\
2016 .\end{array}$ & Transversal & $\begin{array}{l}\text { Local: Tailândia } \\
\text { Amostra: } 600\end{array}$ & $\begin{array}{l}\text { Objetivo: Estudo avaliou a relação entre o letramento em saúde, } \\
\text { aderência à medicação e nível de pressão arterial sistêmica (PAS) } \\
\text { em pacientes hipertensivos. }\end{array}$ \\
\hline
\end{tabular}




\begin{tabular}{|l|l|l|}
\hline & participantes. & $\begin{array}{l}\text { Desfecho: Letramento em saúde elevado foi associado a menores } \\
\text { níveis de pressão arterial. }\end{array}$ \\
\hline
\end{tabular}

Source: Autores (2019).

Os artigos analisados investigaram a associação entre o letramento em saúde e diversos desfechos em saúde da população idosa, três artigos abordaram essa relação com a hipertensão arterial sistêmica (Costa et al. 2019; Shi et al. 2017; Wannasirikul et al. 2019), cinco abordaram essa relação com a diabetes (Santos \& Portella, 2016; Souza et al. 2020; Lamar et al. 2018; Fernández-Silva et al. 2018; Finbraten et al. 2020), um abordou essa relação com a doença pulmonar obstrutiva crônica (Puente-Maestu et al. 2016), um abordou essa relação com a dor e incapacidade (Murtaugh et al. 2017), quatro abordaram essa relação com a insuficiência cardíaca (Fabbri et al. 2018; León-Gonzáles et al. 2017; Como, 2018; Cox et al. 2016) e cinco abordaram essa relação com a saúde neurológica e mental (Yu, Wilson, Han et al. 2017; Do et al. 2020; Liu et al. 2018; Wilson et al. 2017; Yu, Wilson, Schneider et al. 2017).

Desfechos relacionados à hipertensão foram associados ao letramento em saúde em todos os artigos sobre o tema, apresentando baixo LS associado a níveis elevados de pressão arterial sistêmica (PAS) (Costa et al. 2019; Shi et al. 2017; Wannasirikul et al. 2019). Desfechos clínicos relacionados à diabetes foram associados ao letramento em saúde em todos os artigos sobre o tema, apresentando baixo letramento em saúde associado a pior quadro diabético (Santos \& Portella, 2016; Souza et al. 2020; Lamar et al. 2018; Fernández-Silva et al. 2018) e associação entre o letramento em saúde e melhores condições gerais de saúde (Finbraten et al. 2020).

Desfechos clínicos associados a dor e incapacidade não foram associados ao letramento em saúde (Murtaugh et al. 2017). Quanto a associação a doença pulmonar obstrutiva crônica, a afecção foi associada a maior necessidade de assistência em saúde de terceiros, com associação com maior morbimortalidade e pior expectativa de vida (Puente-Maestu et al. 2016). Nos artigos que investigaram a relação com a insuficiência cardíaca, verificou-se associação entre elevado letramento em saúde e menor grau de comprometimento cardíaco (León-Gonzáles et al. 2017), entretanto, um estudo não encontrou nenhuma associação entre o letramento em saúde e desfechos relacionados à insuficiência cardíaca (Como, 2018), quanto a mortalidade, um artigo verificou associação entre LS baixo com risco aumentado de morte (Fabbri et al. 2018); entretanto; em outro trabalho, nenhum nível de LS foi associado com causas de mortalidade (León-Gonzáles et al. 2017).

Quanto a associação do letramento em saúde com a saúde neurológica e mental, verificou-se associação entre o letramento em saúde elevado e menor risco de desenvolvimento de demência por doença de Alzheimer (Wilson et al. 2017), associação entre o baixo letramento em saúde e maior risco de desenvolvimento de demência por doença de Alzheimer (Yu, Wilson, Schneider et al. 2017) e associação entre o rápido declínio do letramento em saúde e maior risco de demência por doença de Alzheimer (Yu, Wilson, Han et al. 2017); a associação com desfecho depressivo e o letramento em saúde foi positiva (Do et al. 2020, Puente-Maestu et al. 2016), entretanto um artigo não identificou relação entre o letramento em saúde e saúde mental (Liu et al. 2018; Como, 2018).

A associação entre letramento em saúde e necessidade de admissão em ambiente ambulatorial ou hospitalar foi identificada em idosos com doença pulmonar obstrutiva crônica (Puente-Maestu et al. 2016) e em idosos com insuficiência cardíaca (Fabbri et al. 2018; Cox et al. 2016).

\section{Discussão}

O letramento em saúde tem relação íntima com a escolaridade e a idade dos pacientes, sendo um determinante social importante no contexto da saúde (Machado et al. 2015). A autopercepção dos idosos, quanto a seu contexto social e clínico, apresenta influência nas atividades de autocuidado praticadas, dessa forma, letramento em saúde apresenta-se como um fator a 
ser considerado na avaliação geral do contexto saúde-doença vivido pelo idoso, sendo agente essencial na avaliação clínica realizada pelos profissionais de saúde, pois quando subestimado, pode contribuir com o agravo dos problemas de saúde apresentados pelos idosos (Romero, Scortegagna \& Doring, 2018).

Os artigos selecionados nesta revisão indicam que o letramento em saúde tem associação direta com variadas formas de desfechos clínicos relacionados a condições gerais e específicas de saúde, salientando que dos artigos selecionados, dois não encontraram nenhuma associação entre as afecções pesquisadas e o letramento em saúde (Como, 2018; Murtaugh et al. 2017).

Hipertensão arterial sistêmica é uma condição crônica comum na terceira idade, atinge mais de $75 \%$ dos indivíduos com mais de 70 anos de idade e apresenta reduzidos índices de controle. A atenção primária é a porta de entrada concomitante da prevenção e do tratamento (Ministério da Saúde, 2014). A associação entre o LS e o inadequado controle da pressão artéria sistêmica é um fenômeno global, ocorrendo em locais com diferentes níveis socioeconômicos, particularidades culturais e prevalência da PAS (Costa et al. 2019). Estudos realizados na Tailândia e na China associou letramento em saúde aos níveis pressóricos dos idosos avaliados, sendo menor letramento em saúde associado a pressão arterial sistólica mais elevada; quanto a adesão à medicação, letramento em saúde também apresentou associação positiva (Wannasirikul et al. 2016; Shi et al. 2017). Em um grupo de pacientes de idosos com HAS, acompanhados em unidades de atenção primária no Brasil, o controle inadequado da PAS foi associado ao LS baixo, sendo o letramento, um fator mais importante que características consagradas do processo patológico da hipertensão arterial sistêmica (HAS) como cor e peso (Costa et al. 2019).

Diabetes mellitus é uma condição crônica prevalente em pessoas idosas. No mundo desenvolvido, é uma doença que figura entre as principais causas de mortalidade na população. No Brasil, atinge 21,6\% dos indivíduos com mais de 65 anos de idade, sendo a faixa etária com maior incidência da doença. Associada a hipertensão arterial sistêmica, figura como a primeira causa de internações hospitalares e óbitos no Sistema Único de Saúde, causando pesados gastos para o sistema de saúde (Ministério da Saúde, 2013). No Brasil, Espanha e Estados Unidos, estudos analisaram a associação do letramento em saúde ao nível do quadro diabético apresentado pelos pacientes idosos, sendo relacionado, independentemente, ao nível de hemoglobina glicada (Souza et al. 2020; Lamar et al. 2018; Fernández-Silva et al. 2018) e a ocorrência de complicações relacionadas a diabetes, como complicações visuais e doença renal (Santos \& Portela, 2016). Apoio social foi fator modificador da relação entre o letramento em saúde e o nível de hemoglobina glicada, podendo alterar o nível de letramento em saúde e favorecer ou comprometer o controle da diabetes (Souza et al. 2020). Estudo realizado na Noruega, não encontrou associação direta entre o controle glicêmico e o letramento em saúde, entretanto, verificou-se que o letramento em saúde está associado a maior autopercepção de empoderamento frente a doença e a melhor condição geral de saúde (Finbraten et al. 2020).

Risco cardiovascular foi verificado por dois artigos selecionados, apresentando elevado risco cardiovascular quando relacionado a baixo letramento em saúde em paciente com hipertensão arterial sistêmica (Shi et al. 2017) e não apresentando relação com o letramento em saúde em pacientes com diabetes mellitus (Fernández-Silva et al. 2018).

Doença pulmonar obstrutiva crônica é uma afeç̧ão crônica respiratória possível de tratamento, mas não completamente reversível, é definida como uma doença que apresenta limitação do fluxo aéreo, de caráter progressivo e com resposta imunológica atípica, apresentando prevalência estimada em 12\% da população maior de 40 anos (Sociedade Brasileira de Pneumologia e Tisiologia, 2004). Doença pulmonar obstrutiva crônica foi positivamente associada ao letramento em saúde, com portadores da afeç̧ão, apresentando maior risco de desenvolvimento de comorbidades concomitantes, quando diagnosticados com letramento em saúde inadequado; esta associação também foi verificada na relação dos pacientes que necessitam de assistência de terceiros, estes pacientes apresentaram menor independência quando verificado letramento em saúde inadequado; além de apresentarem maior morbimortalidade e pior expectativa de vida que os pacientes portadores de doença pulmonar obstrutiva crônica com letramento em saúde adequado (Puente-Maestu et al. 2016). 
Insuficiência cardíaca é uma afecção clínica que acomete o órgão cardíaco, impossibilitando-o de bombear sangue de forma satisfatória e suficiente para suprir a demanda corporal, obrigando-o a aumentar a pressão de enchimento; é considerada uma patologia grave, com significativa prevalência na população idosa, alcançando 17,4\% dos indivíduos com mais de 85 anos de idade (Sociedade Brasileira de Cardiologia, 2018). A relação entre o letramento em saúde e a insuficiência cardíaca foi encontrada em três artigos selecionados nesta revisão, um artigo verificou essa associação na proporção de comorbidades associadas, segundo o estudo, pacientes com insuficiência cardíaca e com letramento em saúde inadequado, apresentaram maior chance de apresentar doenças cerebrovasculares, doenças vasculares periféricas, doença renal e diabetes que paciente com letramento em saúde adequado (Fabbri et al. 2018); outro artigo encontrou associação entre alto letramento em saúde e apresentação de menores níveis de comprometimento cardíaco quando comparado com pacientes com baixo letramento em saúde (León-Gonzalez et al. 2017); já o terceiro artigo encontrou associação entre o baixo letramento em saúde e a necessidade de buscar cuidados de saúde especializados em pacientes com insuficiência cardíaca (Cox et al. 2016). Dois artigos investigaram a relação do letramento em saúde e o aumento da mortalidade em pacientes com insuficiência cardíaca, o resultado apresentado pelos trabalhos foi antagônico, sendo o letramento em saúde inadequado associado a um aumento de 2 vezes no risco de morte quando comparado a uma amostra com letramento em saúde adequado (Fabbri et al. 2018); enquanto o letramento em saúde não apresentou nenhuma associação com as causas de mortalidade em pacientes com a afecção (LeónGonzález et al. 2017).

Idosos que necessitam de hospitalização apresentam redução da qualidade de vida e de suas capacidades; quando comparados os momentos da admissão e da alta, há redução da capacidade de se expressar, redução na mobilidade com aumento da necessidade de ajuda de terceiros e aumento do número de incapazes após a alta hospitalar; dessa forma, a hospitalização está associada à maior nível de dependência e perda da qualidade de vida nos idosos (Santos \& Souza, 2013). A associação entre a necessidade de hospitalização e o letramento em saúde foi identificada em três artigos selecionados pela revisão. Em pacientes com doença pulmonar obstrutiva crônica, o letramento em saúde inadequado foi associado a maior dependência dos pacientes quanto à ajuda de terceiros e a maiores chances de admissões ou visitas ao departamento de emergência (Puente-Maestu et al. 2016). Em pacientes com insuficiência cardíaca, o letramento em saúde inadequado foi associado a um risco maior de internações, apresentando 50\% mais chances de hospitalização em comparação a pacientes com letramento em saúde adequado (Fabbri et al. 2018), além de ser associado a uma maior chance de readmissões em instituições que fornecem cuidados de saúde, na população de pacientes portadores de insuficiência cardíaca que necessitam utilizar atendimento do serviço de saúde nos últimos 30 dias (Cox et al. 2016).

O declínio cognitivo leve é caracterizado como uma diminuição da capacidade cognitiva maior que a diminuição já esperada para a idade e nível educacional do idoso, entretanto, essa redução não pode causar interferência significativa nas atividades cotidianas. O declínio cognitivo leve aumenta o risco de o idoso desenvolver formas de demência conforme envelhece, sendo a demência por doença de Alzheimer a mais relacionada (Rabelo, 2009). Em pacientes com insuficiência cardíaca, foi identificado associação entre o letramento em saúde elevado e menor comprometimento cognitivo (LeónGonzáles et al. 2017). Letramento em saúde elevado também foi associado a menor risco de desenvolvimento de declínio cognitivo leve, além de ser relacionado a apresentação de nível basal de cognição mais elevado (Wilson et al. 2017). Em pacientes idosos foi verificada associação entre a diminuição do letramento em saúde com maior risco de desenvolvimento de declínio cognitivo leve, pois, pacientes que com o envelhecimento apresentaram redução rápida dos escores de letramento em saúde, exibiram maior risco de desenvolver declínio cognitivo leve que idosos que apresentavam redução lenta dos escores de letramento em saúde; já pacientes que previamente obtiveram diagnóstico de declínio cognitivo leve, quando comparados a pacientes idosos de mesma idade sem a afecção, apresentavam menor letramento em saúde (Yu. Wilson, Han et al. 2017). 
Dor e incapacidade física estão relacionadas a limitação na realização das atividades cotidianas em idosos, com restrição às atividades domésticas, lazer e obrigações do dia a dia (Figueiredo, Pereira, Ferreira, Pereira \& Amorim, 2013). Esta revisão selecionou um artigo sobre a relação da dor e perda de função; não foi encontrada associação entre o letramento em saúde e a intensidade da dor referida, também não foi encontrada associação entre o letramento em saúde e a prevalência de incapacidade relacionadas a dor (Murtaugh et al. 2017).

A doença de Alzheimer é uma afecção crônica de comprometimento neurodegenerativo, causando perecimento da memória e de diversos outros campos cognitivos; afeta diretamente a capacidade de realização das atividades de vida diárias de seus portadores; é a causa mais relacionada à demência em pacientes idosos, apresentando prevalência da forma grave em um quinto dos idosos afetados (Sociedade Brasileira de Geriatria e Gerontologia, 2011). Letramento em saúde em idosos foi associado a incidência de demência em três artigos selecionados pela revisão. Declínio do letramento em saúde é um processo natural da senescência, o aumento da velocidade do declínio do letramento em saúde foi associado a maior risco de desenvolvimento de demência por doença de Alzheimer, sendo que a cada aumento de 1\% na taxa de declínio do letramento em saúde, o risco de demência pela afecção aumentou 3 vezes (Yu, Wilson, Han et al. 2017). O alto nível de letramento em saúde foi correlacionado à diminuição do risco de desenvolvimento da afecção (Wilson et al. 2017). A menor alfabetização em saúde foi associada a maior risco de desenvolvimento de demência por doença de Alzheimer; além de apresentar maior carga patológica nos idosos portadores da afecção, mesmo após o controle da cognição (Yu, Wilson, Schneider et al. 2017).

Depressão é uma doença pertencente ao campo dos transtornos psiquiátricos, muito frequente em pacientes idosos; têm importante relação com a qualidade de vida, sendo um relevante problema de saúde; apresenta estreita relação com outros agravos clínicos, tendo seu diagnóstico e tratamento realizado no âmbito da atenção básica, sendo importante a participação dos profissionais de saúde na abordagem inicial e rastreio da afecção na população idosa (Ramos et al. 2019). Quatro estudos selecionados pela revisão avaliaram a relação do letramento em saúde com a saúde mental de pacientes idosos; dois não encontraram correlação entre o nível de letramento em saúde e o estado de saúde mental dos pacientes (Como, 2018; Liu et al. 2018). Entretanto, letramento em saúde inadequado foi associado a maior risco de desenvolvimento de ansiedade e depressão em pacientes idosos com doença pulmonar obstrutiva crônica (Puente-Maestu et al. 2016); além de ser verificado associação entre letramento em saúde elevado e menor risco de depressão em idosos que apresentavam sintomas de COVID-19 (Do et al. 2020).

A importância do letramento em saúde na população idosa pôde ser verificada nos mais diversos desfechos clínicos discutidos, as informações apresentadas nesta revisão de escopo podem servir de auxílio para profissionais de saúde compreenderem a importância de uma abordagem individualizada ao paciente idoso, sendo fonte para desenvolvimento de estratégias direcionadas ao público idoso, que busquem aumentar o letramento em saúde desta população.

Destaca-se as limitações desta pesquisa, devido a insuficiência de trabalhos que apresentem o conceito do letramento em saúde associado a variados desfechos clínicos no público idoso; considerando que a população da terceira idade apresenta múltiplas afecções clínicas que não puderam ser abordadas na presente revisão.

\section{Considerações Finais}

A ausência de letramento adequado é uma adversidade coletiva, contudo, na saúde da população idosa se torna essencial a consideração do nível de LS durante as avaliações clínicas, realizadas pelos profissionais de saúde, pois se torna uma ferramenta relevante para o enfrentamento dos desfechos clínicos relacionados às afecções apresentadas por essa população. Os resultados reforçam a associação do LS com os mais variados desfechos em saúde da população idosa, envolvendo, principalmente, hipertensão arterial sistêmica, diabetes mellitus, insuficiência cardíaca, risco cardiovascular, 
doença pulmonar obstrutiva crônica, internamentos e hospitalizações, declínio cognitivo leve, dor e incapacidade, doença de Alzheimer e depressão.

Observou-se que a associação do letramento em saúde com os mais diversos resultados em saúde na população idosa, apresentou-se como fator de mudança na condição clínica, afetando diretamente os resultados apresentados pelo paciente e a condução e manejo da afecção.

Frente a expressiva importância dos conceitos apresentados, espera-se que as informações expostas por esta revisão, possam fomentar o debate entre os profissionais e gestores de saúde, auxiliando na elaboração de medidas que visem compreender o grau de letramento em saúde da população atendida, objetivando a edificação de ações que contribuam positivamente para as condições de saúde deste público.

Recomenda-se novos estudos para avaliação dos múltiplos desfechos clínicos relacionados à população idosa, buscando investigar a associação do letramento em saúde com outras afecções de saúde, em vista da grande heterogeneidade do complexo saúde-doença.

\section{Referências}

Baker, D. W., Williams, M. V., Parker, R. M., Gazmararian, J. A., Nurss, J. Development of a brief test to measure functional health literacy. (1999) Journal Patient Educ. Couns, 38 (1), 33-42. https://doi.org/10.1016/s0738-3991(98)00116-5.

Carthery-Goulart, M. T., Anguinah, R., Areza-Fegyveres, R., Bahia, V. S., Brucki, S. M. D., Damin, A., Formigoni, A. P., Frota, N., Guariglia, C., Jacinto, A. F., Kato, E. M., Lima, E. P., Mansur, L., Moreira, D., Nóbrega, A., Porto, C. S., Senaha, M. L. H., Silva, M. M., Smid, J., Souza-Talarico, J., Radanovic, M., Nitrini, R. (2009) Performance of a Brazilian population on the test of functional health literacy in adults. Rev. Saúde Pública. 43 (4), 631-638. https://doi.org/10.1590/S0034-89102009005000031.

Como, J. M. (2018) Health Literacy and Health Status in People With Chronic Heart Failure. Clinical Nurse Specialist. Jan/fev. http://dx.doi.org/10.1097/NUR.0000000000000346.

Costa, V. R. S., Costa, P. D. R., Nakano, E. Y., Apolinário, D., \& Santana, A. N. C. (2019) Alfabetismo funcional em saúde em pessoas idosas hipertensas na atenção primária. Revista Brasileira de Enfermagem. 72 (2), 278-285. http://dx.doi.org/10.1590/0034-7167-2018-0897.

Cox, S. R., Liebl, M. G., McComv, M. N., Chau, J. Q., Wilson, A. A., Achi, M., Garey, K. W., \& Wallace, D. (2016) Association between health literacy and 30-day healthcare use after hospital discharge in the failure population. Research in social and Administrative Pharmacy. 1-5. http://dx.doi.org/10.1016/j.sapharm.2016.09.003.

Do, B. N., Nguyen, P., Pham, K. M., Nguyen, H. C., Nguyen, M. H., Tran, C. Q., Nguyen, T. T. P., Tran, T. V., Pham, L. V., Tran, K. V., Duong, T. T., Duong, T. H., Nguyen, K. T., Pham, T. M., Hsu, M., \& Duong, T. V. (2020) Determinants of Health Literacy and Its Associations With Health-Related Behaviors, Depression Among the Older People With and Without Suspected COVID-19 Symptoms: A Multi-Institutional Study. Frontiers in Public Health. 8. https://doi.org/10.3389/fpubh.2020.581746.

Fabbri, M., Yost, K., Rutten, L. J. F., Jensen, D., Weston, S. A., Jiang, R., \& Roger, V. L. (2018) Health Literacy and Outcomes in Patients With Heart Failure: A Prospective Community Study. Mayo Clinic Proceedings. 93 (1), 9-15. https://doi.org/10.1016/j.mayocp.2017.09.018.

Fernández-Silva, M. J., Alonso-González, A., González-Pérez, E., Gestal-Otero, J. J., \& Díaz-Grávalos, G. J. (2019) Alfabetización em salud en pacientes com diabetes tipo 2: um estúdio transversal com el cuestionario HLS-EU-Q47. Sociedad Española de Médicos de Atención Primaria. https://doi.org/10.1016/j.semerg.2018.08.003.

Figueiredo, V. F., Pereira, L. S. M., Ferreira, P. H., Pereira, A. M., \& Amorim, J. S. C. (2013) Incapacidade funcional, sintomas depressivos e dor lombar em idosos. Fisioter Mov. 26 (3). 549-557. https://doi.org/10.1590/S0103-51502013000300008.

Filho, P. P. S., \& Massi, G. A. (2014) Letramento de idosos brasileiros acima de 65 anos. Distúrbios de Comunicação, 26 (2), 267-276. https://revistas.pucsp.br/dic/article/viewFile/16513/14642.

Finbraten, H. S., Gutterrud, O., Nordstrom, G., Pettersen, K. S., Trollvik, A., \& Wilde-Larsson, B. (2020) Explaining variance in health literacy among people with type 2 diabetes: the association between health literacy and health behaviour and empowerment. BMC Public Health. https://doi.org/10.1186/s12889-0208274-z.

Geib, L. T. C. (2012) Determinantes sociais da saúde do idoso. Revista ciência \& saúde coletiva, 17 (1), 123-133. https://doi.org/10.1590/S141381232012000100015 .

IBGE - Instituto Brasileiro de Geografia e Estatística (2016) Síntese de indicadores sociais: Uma análise das condições de vida da população brasileira. IBGE. Rio de Janeiro. https://biblioteca.ibge.gov.br/visualizacao/livros/liv101629.pdf.

Lamar, M., Wilson, R. S., Yu, L., James, B. D., Stewart, C. C., Bennett, D. A., \& Boyle, P. A. (2018) Associations of literacy with diabetes indicators in older adults. Journal Epidemiology Community Health. 0-6. http://dx.doi.org/0.1136/jech-2018-210977. 
León-Gonzáles, R., García-Esquinas, E., Paredes-Galán, E., Ferreto-Martínez, A. I., Gonzaléz-Guerrero, J., Hornillos-Calvo, M., Menéndez-Colino, R., Torres-Torres, I., Galán, M. C., Torrente-Carballido, M., Olzcoz-Chiva, M., Rodríguez-Pascual, C., \& Rodríguez-Artalejo, F. (2017) Health Literacy and Heath Outcomes in Very Old Patients With Heart Failure. Revista Española de Cardiología. http://dx.doi.org/10.1016/j.rec.2017.06.010.

Lima, J. P., Abreu, D. P. G., Bandeira, E. O., Brum, A. N., Mello, M. C. V. A., Varela, V. S., \& Martins, N. F. F. (2019) Letramento Funcional em Saúde e Fatores Associados em Pessoas Idosas. Cogitare enfermagem, 24. http://dx.doi.org/10.5380/ce.v24i0.63964.

Liu, Y., Xue, L., Xue, H., \& Hou, P. (2018) Health Literacy, Physical and Mental Health, and Activities of Daily Living Among, Older Chinese Adults in Nursing Homes. Asia Pacific Journal of Public Health. 30 (6). 592-599. https://doi.org/10.1177/1010539518800368.

Machado, A. L. G., Gubert, F. A., Pinheiro, P. N. C., Vieira, F. C., Oliveira, P. S., \& Guedes, H. (2015) Letramento em saúde e Envelhecimento: Foco em condições crônicas de saúde. Atas - Investigação Qualitativa em Saúde. 2. https://proceedings.ciaiq.org/index.php/CIAIQ/article/view/520.

Maragno, C. A. D., \& Luiz, P. P. V. (2016) Letramento em saúde e adesão ao tratamento medicamentoso: uma revisão da literatura. Revista Iniciação Científica, 14 (1). http://periodicos.unesc.net/iniciacaocientifica/article/viewFile/2672/2480.

Marques, S. R. L., Escarce, A. G., \& Lemos, S. M. A. (2018) Letramento em saúde e autopercepção de saúde em adultos usuários da atenção primária. CoDAS, 30 (2). https://doi.org/10.1590/2317-1782/20182017127.

Massi, G., Torquato, R., Guarinello, A. C., Berberian, A. P., Santana, A. P., \& Lourenço, R. C. (2010) Práticas de letramento no processo de envelhecimento. Revista brasileira de geriatria e Gerontologia, 13 (1), 59-71. https://doi.org/10.1590/S1809-98232010000100007.

Ministério da Saúde. Secretaria de Atenção à Saúde. Departamento de Atenção Básica. (2013) Cadernos de Atenção Básica: Estratégias para o cuidado da pessoa com doença crônica - diabetes mellitus. Brasília: Ministério da Saúde. https://bvsms.saude.gov.br/bvs/publicacoes/estrategias_ cuidado_pessoa_diabetes_mellitus_cab36.pdf.

Ministério da Saúde. Secretaria de Atenção à Saúde. Departamento de Atenção Básica. (2014) Cadernos de Atenção Básica: Estratégias para o cuidado da pessoa com doença crônica - hipertensão arterial sistêmica. Brasília: Ministério da Saúde. https://bvsms.saude.gov.br/bvs/publicacoes/hipertensao_arterial_ sistemica_cab37.pdf.

Morais, E. N. (2012) Atenção à saúde do Idoso: Aspectos Conceituais. Brasília. Publicação: Organização Pan-Americana da Saúde. https://apsredes.org/pdf/Saude-do-Idoso-WEB1.pdf.

Moreira, L. B., Silva, S. L. A., Castro, A. E. F., Lima, S. S., Estevam, D. O., Freitas, F. A. S., Vieira, E. L. M., \& Pereira, D. S. (2020) Fatores associados a capacidade funcional de idosos adscritos à Estratégia de Saúde da Família. Revista ciência \& saúde coletiva, 25 (6), 2041-2050. https://doi.org/10.1590/141381232020256.26092018 .

Murphy, P. W., Davis, T. C., Long, S. W., Jackson, R. H \& Decker, B., C. (1993) Rapid Estimate of Adult Literacy in Medicine (REALM): A Quick Reading Test for Patients. Journal of Reading. 37 (2), 124-130. https://www.jstor.org/stable/40033408.

Murtaugh, C. M., Beissner, K. L., Barrón, Y., Trachtenberg, M. A., Bach, E., Henderson, C. R., Sridharan, S., \& Reid, M. C. (2017) Pain and Funcion in Home Care: A Need for Treatment Tailoring to Reduce Disparities? Clinical Pain. 33 (4). https://doi.org/10.1097/AJP.0000000000000410.

Neto, J. A. C., Costa, L. A., Estevanin, G. M., Bignoto, T. C., Vieira, C. I. R., Pinto, F. A. A. R., \& Ferreira, R. E. (2019) Letramento funcional em saúde nos portadores de doenças cardiovasculares crônicas. Revista Ciência e Saúde Coletiva, 24 (3), 1121-1132. https://doi.org/10.1590/1413-81232018243.02212017.

Parker, R. M., Baker, D. W., Williams, M. V., Nurss, J. R. (1995) The Test of Functional Health Literacy in Adults: A New instrument for Measuring Patients' Literacy Skills. J. Gen. Intern. Med. 10, 537-541. https://www.doi.org/10.1007/BF02640361.

Puente-Maestu, L., Calle, M., Rodríguez-Hermosa, J. L., Campuzano, A., Díez, J. M., Álvarez-Sala, J. L., Puente-Andues, L., Pérez-Gutiérrez, M. J., \& Lee, S. D. (2016) Health literacy and health outcomes in chronic obstructive pulmonary disease. Respiratory Medicine, 115, 78-82. https://doi.org/10.1016/j.rmed.2016.04.016.

Rabelo, D. F. (2009) Comprometimento Cognitivo Leve em Idosos: avaliação, fatores associados e possibilidades de intervenção. Revista Kairós Gerontologia. 12 (2). 65-79. https://revistas.pucsp.br/index.php/kairos/article/view/4414.

Ramos, F. P., Silva, S. C., Freitas, D. F., Gangussu, L. M. B., Bicalho, A. H., Sousa, B. V. O., Rametta, Z. M. J., Rametta, F. J., Rametta, F. J., Rametta, L. P. M., Nascimento, C. I. C., Santos, S. H. S., \& Guimarães, T. A. (2019) Fatores associados à depressão em idoso. Revista eletrônica Acervo Saúde. https://doi.org/10.25248/reas.e239.2019.

Romero, S. S., Scortegagna, H. M., Doring, M. (2018) Nível de Letramento Funcional em Saúde e Comportamento em Saúde de Idosos. Texto Contexto Enfermagem. 27 (4). http://dx.doi.org/10.1590/0104-07072018005230017.

Santos, G. \& Souza, L. (2013) Qualidade de vida em pessoas idosas hospitalizadas: comparação da admissão com a alta do internamento. Revista Kairós Gerontologia. 16 (2). 7-25. https://revistas.pucsp.br/index.php/kairos/article/view/17625.

Santos, M. I. P., \& Portella M. R. (2016) Condições do letramento funcional em saúde de um grupo de idosos diabéticos. Revista Brasileira de Enfermagem, 69 (1), 156-164. http://dx.doi.org/10.1590/0034-7167.2016690121i.

Santos, M. I. P., Portella, M. R., Scortegagna, H. M., \& Santos. P. C. S. (2015) Letramento funcional em saúde na perspectiva da enfermagem Gerontológica: revisão integrativa da literatura. Revista Brasileira de Geriatria e Gerontologia, 18 (3), 651-664. http://dx.doi.org/10.1590/1809-9823.2015.14080.

Shi, D., Li, J., Wang, Y., Liu, K., Shi, R., Zhang, Q., \& Chen, X. (2017) Association between health literacy and hipertensión management in a Chinese community: a retrospective cohort study. Intern Emerg Med. http://dx.doi.org/10.1007/s11739-017-1651-7.

Sociedade Brasileira de Cardiologia (2018) Diretriz Brasileira de Insuficiência Cardíaca Crônica e Aguda. Arquivos Brasileiro de Cardiologia. 111 (3). 436539. http://dx.doi.org/10.5935/abc.20180190. 
Research, Society and Development, v. 10, n. 11, e495101119726, 2021

(CC BY 4.0) | ISSN 2525-3409 | DOI: http://dx.doi.org/10.33448/rsd-v10i11.19726

Sociedade Brasileira de Geriatria e Gerontologia (2011) Doença de Alzheimer: Prevenção e Tratamento. Diretrizes Clínicas na Saúde Suplementar. Rio de Janeiro. https://sbgg.org.br/wp-content/uploads/2014/10/alzheimer-prevencao.pdf.

Sociedade Brasileira de Pneumologia e Tisiologia (2004) II Consenso Brasileiro sobre Doença Pulmonar Obstrutiva Crônica - DPOC. 30 (5). Brasília. http://www.jornaldepneumologia.com.br/details-supp/40.

Souza, J. G., Farfel, J. M., Jaluul, O., Queiroz, M. S., \& Nery, M. (2020) Associação entre alfabetismo em saúde e controle glicêmico em idosos com diabetes tipo 2 e efeito modificador do suporte social. Einstein. 18. 1-9. http://dx.doi.org/10.31744/einstein_journal/2020AO5572.

The Joanna Briggs Institute (2021) JBI Manual for evidence synthesis. Adelaide, South Australia. The Joanna Briggs Institute. https://wiki.jbi.global/display/MANUAL/JBI+Manual+for+Evidence+Synthesis.

Wannasirikul, P., Termsirikulchai, L., Sujirarat, D., Benjakul, S., \& Tanasugarn, C. (2016) Health literacy, Medication Adherence, and Blood Pressure Level Among Hypertensive Older Adults Treated at Primary Health Care Centers. Southeast Asian Journal Trop Med Public Health. 47 (1). 109-120. https://www.tm.mahidol.ac.th/seameo/2016-47-1/13-65364-109.pdf.

Wilson, R. S., Yu, L., James, B. D., Bennett, D. A., \& Boyle, P. A. (2017) Association of financial and health literacy with cognitive health in old age. Neuropsychol Dev Cogn B Aging Neuropsychol Cogn. 24 (2). 186-197. https://doi.org/10.1080/13825585.2016.1178210.

Yu, L., Wilson, R. S., Han, S. D., Leurgans, S., Bennett, D. A., \& Boyle, P.A. (2017) Decline in Literacy and Incident AD Dementia Among CommunityDwelling Older Persons. Journal of Aging and Health. 1-17. https://journals.sagepub.com/doi/10.1177/0898264317716361.

Yu, L., Wilson, R. S., Schneider, J. A., Bennett, D. A. \& Boyle, P. A. (2017) Financial and Health Literacy Predict Incident Alzheimer's Disease Dementia and Pathology. Journal of Alzheimer's Disease. 56. 1485-1493. https://doi.org/10.3233/JAD-161132. 